\title{
A fast, low perturbation ionization beam profile monitor based on a gas-jet curtain for the ultra low energy storage ring
}

\author{
M. Putignano $\cdot$ K.-U. Kühnel • \\ C.-D. Schröter · C. P. Welsch
}

Published online: 26 August 2009

(C) The Author(s) 2009. This article is published with open access at Springerlink.com

\begin{abstract}
An ionization beam profile monitor relying on a supersonic gas-jet shaped into an extended, thin curtain is proposed for operation in an ultra high vacuum environment for very low perturbation on the accelerated beam, as requested for the in-ring profile monitoring at the Ultra low energy Storage Ring (USR) at the Facility for Low-energy Antiproton and Ion Research (FLAIR), in Darmstadt. In this paper, we describe the working principle of the monitor, as well as providing an analysis of sensitivity and resolution dependence on geometrical design and gas curtain thickness.
\end{abstract}

Keywords Diagnostics • Gas-jet • Profile monitor $\cdot$ Low-perturbation

\section{Introduction}

Low-energy physics and storage rings are recently attracting growing interest in the scientific community, as remarkable characteristics of quantum systems are most conveniently studied at low projectiles energies in the keV range [1,2]. Development of low-energy storage rings causes widespread beam diagnostic technologies to become obsolete. In particular preservation of the beam lifetime causes destructive and even perturbing profile monitoring (interceptive foils) to be ruled out [3]. Furthermore, existing non-perturbing techniques such as residual gas monitors can take up to about $100 \mathrm{~ms}$ [4] to make meaningful measurements, due to the low

M. Putignano $(\varangle) \cdot$ K.-U. Kühnel · C.-D. Schröter

Max Planck Institut für Kernphysik, 69117 Heidelberg, Germany

e-mail: Massimiliano.Putignano@mpi-hd.mpg.de

M. Putignano · C. P. Welsch

Gesellschaft für Schwerionenforschung, 64291 Darmstadt, Germany

C. P. Welsch

Kirchhoff Institut für Physik, Universität Heidelberg, 69120 Heidelberg, Germany 
residual gas pressure, hence not allowing the measurement of a single bunch, at the expected operating pressure of around $10^{-11}$ mbar. A possible solution around these limitations is constituted by a neutral supersonic gas jet target shaped into a thin curtain and bi-dimensional imaging of the gas ions created by impact with the projectiles. Such monitor, as compared to those based on residual gas, allows injection of additional gas (in order to increase the ionization rate) together with efficient evacuation (to keep the required vacuum levels elsewhere in the storage ring), due to the high directionality of the supersonic jet [5]; furthermore, it allows simultaneous determination of both transversal profiles and even beam imaging [6].

In this contribution, we first describe the working principle of a gas-jet curtain monitor and its advantages with respect to residual gas monitors. We then present a study of the intrinsic sensitivity and resolution of the monitor depending on its geometrical design, therewith highlighting the importance of the curtain geometric width.

\section{Beam profile monitor analysis}

\subsection{Working principle}

The proposed beam profile monitor relies on a neutral gas-jet, shaped into a thin curtain and crossing the beam. In its simplest configuration, shown in Fig. 1, the gas curtain flows perpendicularly to the projectiles' propagation axis, and the curtain plane forms with the same axis an angle of $45^{\circ}$. When the projectile beam crosses the gas-jet, ionisation interactions occur and gas ions are created in the region of the curtain. These ions are accelerated by a $5 \mathrm{kV} / \mathrm{m}$ extraction field towards an amplification stage with Micro Channel Plates (MCP) and hence detected via a Position Sensitive Detector (PSD), namely a phosphor screen and a CCD camera. The magnitude of the extraction field is large enough to project the ions on the PSD making negligible the contributions of initial velocity spread. To counterbalance the effects of the extraction field on the main beam, two $3 \mathrm{kV} / \mathrm{m}$ correction fields are added, as shown in Fig. 1. Even at lowest beam energies, this results in a net displacement of the beam from its main orbit of only $1 \mathrm{~mm}$ at the interaction point, and both the displacement and the transverse momentum introduced by the extraction field are fully counterbalanced. After having crossed the beam in the interaction chamber the gas-jet flows into the dumping chamber, where an appropriate vacuum system dumps the jet preventing it from affecting the vacuum in the beam pipes.

The gas-jet consists of a high density curtain shaped region where densities in the range of $10^{10}-10^{13}$ particles $/ \mathrm{cm}^{3}$ can be obtained by varying the stagnation pressure of the jet gas reservoir. Preliminary measurements show that the jet does not sensibly affect the vacuum in a $10^{-11}$ mbar chamber, thanks to its high directionality. If Argon gas is used, for whose ionization by slow antiproton impact cross sections in the order of $10^{-20} \mathrm{~m}^{2}$ have been reported [7], for a $1 \mathrm{~cm}$ curtain (including also the lower pressure region around the main curtain), these densities correspond to a probability of interaction per particle per turn of $10^{-6}$ to $10^{-3}$. Even in the worst case, allowing a hundred collisions before a particle falls out of acceptance, beam lifetimes of $1 \mathrm{~s}$ are still possible in machines such as the USR $(\approx 20 \mu$ s revolution time). For beams of $10^{7}$ particles, these probabilities would in turn lead to a reaction rate in the order 
Fig. 1 Sketch of the gas curtain ionization profile monitor working principle

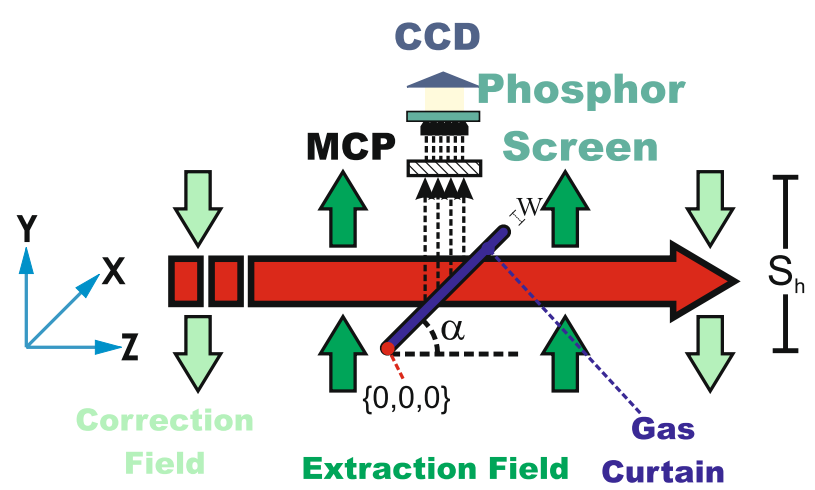

of $10^{5}$ to $10^{8}$ events per second, compatible with ms to $\mu$ s imaging and consequent single $1 \mu$ s bunch measurement.

This profile measurement method allows the actual bi-dimensional imaging of the transverse beam density distribution, hence providing the measured function $\rho(x, y)$. From $\rho(x, y)$, both transverse profiles $\rho_{\text {tot }}(x)$ and $\rho_{\text {tot }}(y)$ can be computed by direct integration of the measured densities:

$$
\rho_{\text {tot }}(y)=\int_{-\infty}^{\infty} \rho(x, y) d x ; \quad \rho_{\text {tot }}(y)=\int_{-\infty}^{\infty} \rho(x, y) d x
$$

This, together with the applicability in ultra high vacuum environment, is another advantage over the ionisation residual gas monitors, which provide only a measure of a single, already integrated transverse profile (either $\rho_{\text {tot }}(x)$ or $\rho_{\text {tot }}(y)$ ); hence two residual gas monitors are needed to measure both profiles, and the combined function $\rho(x, y)$ is not measurable.

\subsection{Sensitivity and precision of the monitor}

To compute the sensitivity and resolution intrinsic to the monitor itself (i.e. without taking into account the extraction fields and the detection system) we analyze the monitor in its simplest configuration, referring again to Fig. 1. A particle travelling along in the $+\mathrm{x}$ direction, and starting at the point $\mathrm{x}, \mathrm{y}, 0$ can ionize a gas atom anywhere in the segment $\left\{\left(x ; y ; \frac{y}{\tan (\alpha)}\right) ;\left(x ; y ; \frac{y}{\tan (\alpha)}+\frac{w}{\sin (\alpha)}\right)\right\}$ which would in turn result in a projection on the position sensitive detector in the segment $\left\{\left(x ; S_{h} ; \frac{y}{\tan (\alpha)}\right) ;\left(x ; S_{h} ; \frac{y}{\tan (\alpha)}+\frac{w}{\sin (\alpha)}\right)\right\}$. An effective width of the curtain can then be defined as the distance travelled by a projectile through the gas in a straight line, i.e. $w / \sin (\alpha)$. Referring with the subscript $s$ to the coordinates of the image on the sensor and with the subscript $i$ to the initial coordinates of the ionizing particle, the sensitivities of the profile monitor for each direction become:

$$
S_{x}=\frac{d x_{s}}{d x_{i}}=1=M_{x} ; \quad S_{y}=\frac{d z_{s}}{d y_{i}}=\tan (\alpha)^{-1}=M_{y},
$$

which also represent the magnification Mx and My of the beam profile's image on the position sensitive detector. The precision can instead be calculated considering the influence of the uncertainty due to the curtain width on the position of ionization. 
This influences only the vertical (y axis) profile, introducing a flat error distribution with a full width of $w / \sin (\alpha)$. This in turn results in an intrinsic final resolution in the $\mathrm{y}$ direction poorer than in the $\mathrm{x}$ direction, as it has also been reported in the work of Hashimoto [5], where much effort has been devoted to decreasing the curtain width. It must be noticed that a more correct indication of the precision should take into account the magnification of the beam profile image on the detector.

We can introduce the modified error distribution full width $W_{E r r}$ scaled with the value of magnification:

$$
W_{E r r, y}=\frac{(w / \sin (\alpha))}{S_{y}}=\frac{w}{\cos (\alpha)}
$$

Whilst it is in principle possible to minimize the $W_{E r r}$ by decreasing the value of $\alpha$, hence effectively improving the resolution, Eq. 3 shows, however, that it is possible to gain only a factor $\frac{2}{\sqrt{2}}$ as compared to the $45^{\circ}$. case. The value of $\alpha$ is then rather chosen in order to have a value of magnification equal in the $\mathrm{x}$ and $\mathrm{y}$ direction, leading to a non-deformed image, thus avoiding the need of image post-processing. As the $\mathrm{x}$-axis magnification is equal to unity and independent from the value of $\alpha$, the $\mathrm{y}$-axis magnification is also chosen to be unity, corresponding to $\alpha=45^{\circ}$. It is now clear how the extent of the vertical resolution degradation depends only on the width of the curtain, which becomes a factor of primary concern in the design of the nozzleskimmer system used for the creation of the gas-jet.

\section{Conclusions}

The working principle and estimated performances of a non-perturbing ionization beam profile monitor based on a gas-jet curtain have been presented and the monitor itself proposed for beam diagnostics in ultra low energy, ultra high vacuum storage rings. As compared to existing non-perturbing residual gas profile monitors, the proposed system provides higher count rates, easier additional gas evacuation and beam imaging (hence simultaneous determination of both transverse profiles). The design of the nozzle-skimmer system to be used for shaping the gas-jet into a thin curtain is presently being optimized and will soon be tested experimentally. In parallel, the overall geometry is being optimized by numerical simulations with the GDT code.

Acknowledgements Work supported by GSI and the Helmholtz Association of National Research Centers (HFG) under contract number VH-NG-328.

Open Access This article is distributed under the terms of the Creative Commons Attribution Noncommercial License which permits any noncommercial use, distribution, and reproduction in any medium, provided the original author(s) and source are credited.

\section{References}

1. Welsch, C.P., et al.: FLAIR-A facility for low-energy antiproton and ion research. Hyperfine Interact. 172, 71 (2006)

2. Andersen, L.H., et al.: Physics with electrostatic rings and traps. J. Phys. B: At. Mol. Opt. Phys. 37, R57-R88 (2004) 
3. Harasimowicz, J., et al.: Beam instrumentation for the future ultra-low energy electrostatic storage ring at FLAIR. In: LEAP08 Conference Proceedings (2008)

4. Honma, T., et al.: Design and performance of a non-destructive beam-profile monitor utilizing charge-division method at HIMAC. Nucl. Instrum. Methods A 490(3), 435-443 (2002)

5. Koziol, H.: Beam diagnostics for accelerators. In: Fifth General Accelerator Physics Course, CERN Accelerator School, pp. 565-599 (1994)

6. Hashimoto, Y., et al.: Oxygen gas-sheet beam profile monitor for the synchrotron and storage ring. Nucl. Instrum. Methods Phys. Res. A 527(3), 289-300 (2004)

7. Knudsen, $\mathrm{H}$., et al.: Ionization of atoms and molecules by antiproton impact. Hyperfine Interact. 109, 133 (1997) 